\title{
TAMING THE MOVABLE SINGULARITIES
}

\author{
JARMO HIETARINTA ${ }^{1}$
}

(Received 12 April, 2000)

\begin{abstract}
We have finally obtained for each of the 6 Painlevés an expression of $z, w, w^{\prime}$ that behaves as $1 /\left(z-z_{0}\right)+O(1)$ at each kind of movable singular point. This expression is polynomial in $w^{\prime}$ (at most quadratic), and rational in $w$ and $z$. After it is integrated and exponentiated it yields a function that has a simple zero at each of the singular points.
\end{abstract}

\section{Introduction}

It has been observed many times that the original variables in which an equation is first obtained are perhaps not the best variables for all purposes. The determination of what is "best" depends of course on what aspect of the equation is studied.

A well-known example is provided by the soliton equations. The dependent variable $u$ in the Korteweg-de Vries equation $u_{x x x}+6 u u_{x}+u_{t}=0$ is natural from the physical point of view as it represents the height of the water wave. However, if we want to construct multi-soliton solutions for this equation they are not particularly simple in terms of $u$. For this purpose one is advised to use a new dependent variable $F$, related to $u$ by

$$
u=2 \partial_{x}^{2} \log F,
$$

because then multi-soliton solutions are expressed as finite polynomials in exponentials. For example, the two-soliton solution of the $\mathrm{KdV}$ equation is given by $F=$ $1+e^{\eta_{1}}+e^{\eta_{2}}+A_{12} e^{\eta_{1}+\eta_{2}}$, where $\eta_{i}=p_{i} x-p_{i}^{3} t+\eta_{i}^{0}$ and $A_{i j}=\left(\left(p_{i}-p_{j}\right) /\left(p_{i}+p_{j}\right)\right)^{2}$. We observe also that $F$ is quite regular everywhere in the complex plane, whereas $u$ computed with (1) has singularities.

\footnotetext{
'Department of Physics, University of Turku, FIN-20014 Turku, Finland; e-mail: Jarmo.Hietarinta@utu.fi.

(C) Australian Mathematical Society 2002, Serial-fee code 1446-1811/02
} 
By the way, the substitution (1) transforms the KdV equation into Hirota form $\left(D_{x}^{4}+D_{x} D_{t}\right) F \cdot F=0$ (after one integration), where $D$ is Hirota's bilinear derivative

$$
D_{z}^{n} A \cdot B=\left.\left(\partial_{z_{1}}-\partial_{z_{2}}\right)^{n} A\left(z_{1}\right) B\left(z_{2}\right)\right|_{z=z_{1}=z_{2}} \text {. }
$$

This fact by itself does not imply that $F$ is an entire function (or a $\tau$-function). An example of this is given in [6]: it is possible to represent the Kaup-Kupershmidt equation $u_{1}+u_{5 x}+30 u u_{3 x}+75 u_{x} u_{2 x}+180 u^{2} u_{x}=0$ as a pair of bilinear equations, starting with the substitution $u=\alpha \partial_{x}^{2} \log G$, where $\alpha=2,1 / 2$ or $1 / 4$, but only with $\alpha=1 / 2$ are the new functions optimal. Thus one must always verify the niceness of the new function. Things are clearcut with solitons because we know the solution explicitly.

Other equations may not have equally simple solutions, but one can still pose the same question: What is a good set of variables? Here our objective is to find new dependent variables, obtained from the solutions of the Painleve equations, such that the new function is as regular as possible, perhaps even an entire function.

Painlevé himself addressed the problem of new regular dependent variables in [4] and stated that since the equations were meromorphic functions they could be represented as a ratio of two entire functions.

Unfortunately Painlevé did not give any derivation for his results, and one can ask whether there is a systematic way to approach this question. In [2] Martin Kruskal and the present author gave a partial solution to this problem, and in [1] the results were further improved, but a uniform treatment of all kinds of movable singularities was still lacking. In [2] we used an ansatz that was polynomial in $w$ and this worked for some equations and expansions (those where the free parameter, in addition to the position of the singularity, was far enough along the expansion). Martin Kruskal urged me to generalize the ansatz to rational expansions, because "it must work".

Here we present a complete solution: for each Painlevé equation we have an expression that behaves as $1 /\left(z-z_{0}\right)$ at each kind of movable singularity. Using it one can construct a function that is entire, except maybe around possible fixed singularities. In other words, the expressions presented here are regular around each and every movable singularity, but the behaviours near fixed singularities (if any) are not known.

\section{The present method}

Let us recall that the equation may become singular for certain values of the dependent variable $w$ or independent variable $z$. The value $w=\infty$ is singular for each Painlevé equation, in addition the value $w=0$ is singular for $P_{\mathrm{III}}-P_{\mathrm{VI}}, w=1$ for $P_{\mathrm{V}}, P_{\mathrm{VI}}$ and $w=z$ for $P_{\mathrm{VI}}$. The dependent variable $w$ may attain this value at any point 
in the $z$-plane depending on the initial values, and the singularity is therefore called movable. By a suitable rational transformation we can take any of the singularities of $w$ into the singularity of type $w=\infty$. In practice we will do this and therefore it is enough to develop the technical aspects of the singularity analysis only for this case.

The fixed singularities of the Painlevé equations are $z=0$ for $P_{\mathrm{III}}, P_{\mathrm{V}}$ and $P_{\mathrm{VI}}$, and $z=1$ for $P_{\mathrm{VI}}$. We do not have anything new to say about the fixed singularities and our results guarantee regular behaviour only at the movable singularities, and therefore the resulting expression is an entire function only provided that the possible fixed singularity does not cause trouble.

The concrete problem for each of the Painleve equations is therefore as follows: Construct an expression of the form

$$
H_{n}\left(w^{\prime}, w, z\right)=A_{n} w^{2}+B_{n} w^{\prime}+C_{n}, \quad A_{n}, B_{n}, C_{n} \text { rational in } w, z
$$

such that

$$
H_{n}=\frac{1}{\left(z-z_{0}\right)^{n}}+O(1), \quad n=1,2
$$

at each type of movable singular point. It is also required that $H_{n}$ does not have a worse kind of singularity somewhere else. [A priori it was not obvious that it would be enough to consider an expansion quadratic in $w^{\prime}$. Since it turns out that $A_{1}$ is always nonzero we cannot just take $H_{2}=H_{1}^{2}$.]

As an example consider $P_{\mathrm{III}}$ and $P_{\mathrm{IV}}$. These equations are singular at $w=\infty$ and $w=0$. The second singularity is transformed to $\infty$ by $w=1 / u$. If this transformation is made both in the equation and in the expression $H_{n}$ we get from the Painlevé equation an expansion for $u$ around its movable singularity (which now is of type $\left.\propto 1 /\left(z-z_{0}\right)\right)$, and the requirement is that when this is substituted in the transformed $H_{n}$ they should again behave as in (3). ${ }^{2}$

The previous results [2] gave very simple $H_{2}$ 's for $P_{1}$ and $P_{\mathrm{II}}$ and therefore here we first tried to construct $H_{2}$ for all Painlevé equations. However, $H_{1}$ is more fundamental, because by taking its derivative we get $-H_{2}$. Since $H_{1}$ must be polynomial at least for $P_{\mathrm{I}}$ and $P_{\mathrm{II}}$ it means that we must consider polynomials that are nominally of higher order in $1 /\left(z-z_{0}\right)$ and for which the leading terms must cancel. The systematic approach is then to increase the order until a result is obtained. Example: for $P_{\mathrm{I}} w$ is of order 2 , the next order 3 can be obtained only with $w^{\prime}$, order 4 only with $w^{2}$, and order 5 only with $w^{\prime} w$, but order 6 both with $w^{2}$ and $w^{3}$. Since the leading orders must cancel we must go at least to order 6 .

\footnotetext{
${ }^{2}$ Note that here usually $w \sim C /\left(z-z_{0}\right)+\cdots$ so a cheap solution would be to take $H_{1}=-w^{\prime} / w \sim 1 /\left(z-z_{0}\right)$, and $e^{-\int\left(w^{\prime} / w\right) d z} \sim z-z_{0}$, but then near a zero of $w$ we would get $-w^{\prime} / w \sim-1 /\left(z-z_{0}\right)$, and therefore $e^{-\int\left(w^{\prime} / w\right) d z} \sim 1 /\left(z-z_{0}\right)$, and thus we would have eliminated one singularity and created another.
} 
As mentioned above it is enough if we can obtain an expression like (2) for $H_{1}$, because then $-d H_{1} / d z$ has the correct expansion for $H_{2}$. In fact

$$
\begin{aligned}
\frac{d}{d z} H_{1} & =w^{\prime \prime} \frac{\partial H_{1}}{\partial w^{\prime}}+w^{\prime} \frac{\partial H_{1}}{\partial w}+\frac{\partial H_{1}}{\partial z} \\
& =\left[w^{\prime \prime}-P\left(z, w, w^{\prime}\right)\right] \frac{\partial H_{1}}{\partial w^{\prime}}+P\left(z, w, w^{\prime}\right) \frac{\partial H_{1}}{\partial w^{\prime}}+w^{\prime} \frac{\partial H_{1}}{\partial w}+\frac{\partial H_{1}}{\partial z}
\end{aligned}
$$

where $P\left(z, w, w^{\prime}\right)$ is the left-hand side of the Painlevé equation. This means that $H_{2}$ defined by

$$
-H_{2}:=P\left(z, w, w^{\prime}\right) \frac{\partial H_{1}}{\partial w^{\prime}}+w^{\prime} \frac{\partial H_{1}}{\partial w}+\frac{\partial H_{1}}{\partial z}
$$

has the behaviour of (3). There may be a problem with respect to (2), because (5) may contain $w^{\prime 3}$ terms, but in fact $A_{1}$ turns out to be such that these terms cancel.

Once the expressions $H_{n}$ have been obtained one can construct a function $F$ by

$$
F:=e^{\int H_{1} d z}=e^{-\iint H_{2} d z d z} \sim\left(z-z_{0}\right)+\text { h.o... }
$$

and $G$ by

$$
G:=w F
$$

and then these functions are regular at the movable singularities of the original solution. The corresponding Painlevé equation can then be expressed in terms of $F$ and $G$ as follows:

$$
\begin{aligned}
(\log F)^{\prime} & =H_{1}\left(z,(G / F),(G / F)^{\prime}\right), \\
(\log F)^{\prime \prime} & =H_{2}\left(z,(G / F),(G / F)^{\prime}\right) .
\end{aligned}
$$

This set of equations is third order, with the third integration constant being related to the scaling invariance $G, F \rightarrow \rho G, \rho F$. After clearing the denominators, this pair of equations is homogeneous in $F, G$ and can in some cases be written in the Hirota bilinear form. ${ }^{3}$ (In [1] it was assumed that $H_{2}$ depends only on $z, w$, see for example (9) and $[1,(4.8)]$.

In the following sections we will go through the Painlevé equations one by one and show how the nonsingular expressions can be constructed.

\section{The results}

3.1. $P_{1}$ : The first Painlevé equation is given by

$$
w^{\prime \prime}=6 w^{2}+z \text {. }
$$

\footnotetext{
${ }^{3}$ The pair (8), (9) is not completely equivalent to the original Painlevé equation, because by (4) $d H_{1} / d z+H_{2}=0$ is possible also if $w$ solves $\partial H_{1} / \partial w^{\prime}=0$.
} 
It has movable singularities where $w \sim \infty$ and the expansion around that is given by

We can therefore take

$$
w=\frac{1}{\left(z-z_{0}\right)^{2}}+O\left(\left(z-z_{0}\right)^{2}\right)
$$

$$
H_{2}^{\mathrm{I}}=w .
$$

If one restricts the ansatz to a polynomial in $w^{\prime}, w, z$ one quickly recovers Painlevé's result [4] for $H_{1}^{\mathrm{I}}$ :

$$
H_{1}^{\mathrm{I}}=\frac{1}{2} w^{2}-2 w^{3}-z w .
$$

As mentioned before, this seems to start as $\left(z-z_{0}\right)^{-6}$ but the leading terms cancel and yield the required behaviour (3). Clearly

$$
\frac{d}{d z} H_{1}^{\mathrm{I}}+H_{2}^{\mathrm{1}}=w^{\prime}\left[w^{\prime \prime}-6 w^{2}-z\right]=0 \text { modulo } P_{\mathrm{I}} .
$$

3.2. $\boldsymbol{P}_{\mathrm{II}}$ : Painlevé's second equation is given by

$$
w^{\prime \prime}=2 w^{3}+w z+\alpha,
$$

and the expansion around any movable singularity is given by

One now finds

$$
w=\frac{ \pm 1}{z-z_{0}}+O\left(z-z_{0}\right) .
$$

$$
H_{1}^{\mathrm{II}}=w^{2}-w^{4}-z w^{2}-2 \alpha w, \quad H_{2}^{\mathrm{II}}=w^{2} .
$$

These results were reported before in [4].

3.3. $P_{\mathrm{III}}$ : The third Painlevé equation is

$$
w^{\prime \prime}=\frac{1}{w} w^{2}-\frac{1}{z} w^{\prime}+w^{3}+\frac{1}{z}\left(\alpha w^{2}+\beta\right)-\frac{1}{w} .
$$

Note that the equation is now singular for $w=\infty$ and $w=0$. Around a movable singularity at $w=\infty$ the solution has the expansion

$$
w= \pm \frac{1}{z-z_{0}}-\frac{\alpha \pm 1}{2 z_{0}}+O\left(z-z_{0}\right) .
$$

In order to analyze the other singularity we transform it to infinity by $w=1 / u$. This yields an equation similar to to (10) except that $\alpha \rightarrow-\beta, \beta \rightarrow-\alpha$, and for $u$ we can therefore use expansion (11) with the same change. 
Since we must now search for an expression that is regular at each singularity the expression may contain negative powers of $w$. The ansatz $\operatorname{Pol}\left(z, w, w^{\prime}\right) / w^{2}$ works and yields

$$
H_{1}^{\mathrm{III}}=\frac{z}{2} \frac{w^{2}}{w^{2}}-\frac{z}{2}\left(w^{2}+\frac{1}{w^{2}}\right)-\alpha w+\frac{\beta}{w}, \quad H_{2}^{\mathrm{III}}=\frac{1}{2} \frac{w^{2}}{w^{2}}+\frac{1}{2}\left(w^{2}+\frac{1}{w^{2}}\right) .
$$

Note that these expressions are manifestly invariant under $w \rightarrow 1 / w$ (and associated parameter changes) and therefore the given asymptotic behaviour is clearly valid for both types of movable singularities: when $w \sim \infty$ and when $w \sim 0$.

3.4. $P_{I V}$ : The fourth Painlevé equation is given by

$$
w^{\prime \prime}=\frac{1}{2 w} w^{2}+\frac{3}{2} w^{3}+4 z w^{2}+2\left(z^{2}-\alpha\right) w+\frac{\beta}{w} .
$$

This equation also has singularities at $w=\infty$ and $w=0$. The expansion around the movable singularity of $w=\infty$ is given by

$$
w=\frac{ \pm 1}{z-z_{0}}-z_{0}+\frac{1}{3}\left[-4 \pm\left(2 \alpha+z_{0}^{2}\right)\right]\left(z-z_{0}\right)+O\left(\left(z-z_{0}\right)^{2}\right) .
$$

The transformation $w=1 / u$ moves the singularity $w=0$ to $u=\infty$ and for $u$ we get a different equation

and expansion

$$
u^{\prime \prime}=\frac{3}{2 u} u^{2}-\beta u^{3}-2\left(z^{2}-\alpha\right) u-4 z-\frac{3}{2 u}
$$

$$
u=\frac{ \pm i / \sqrt{2 \beta}}{z-z_{0}}+O(1)
$$

A computer search with an ansatz as in $P_{\mathrm{ll}}$ yields

$$
\begin{aligned}
& H_{1}^{\mathrm{IV}}=\frac{1}{2} \frac{w^{2}}{w}+\frac{w^{\prime}}{w}-\frac{1}{2} w^{3}-2 z w^{2}-2 w\left(z^{2}-\alpha\right)+\frac{\beta}{w}, \\
& H_{2}^{\mathrm{IV}}=\frac{1}{2} \frac{w^{\prime 2}}{w^{2}}+\frac{1}{2} w^{2}-2\left(z^{2}-\alpha\right)-\frac{\beta}{w^{2}} .
\end{aligned}
$$

(Our previous polynomial result [2] was only applicable to the $w=\infty$ case.)

3.5. $P_{\mathrm{v}}$ : The fifth Painlevé equation

$$
w^{\prime \prime}=\left(\frac{1}{2 w}+\frac{1}{w-1}\right) w^{2}-\frac{1}{z} w^{\prime}+\frac{(w-1)^{2}}{z^{2}}\left(\alpha w+\frac{\beta}{w}\right)+\gamma \frac{w}{z}+\delta \frac{w(w+1)}{w-1}
$$


has three singular points, $w=0,1, \infty$. Around $w=\infty$ the expansion is

$$
w= \pm \frac{z_{0} / \sqrt{2 \alpha}}{z-z_{0}}+O(1) .
$$

The singularity $w=0$ is transformed to $u=\infty$ by $w=1 / u$, and the resulting equation and expansion are as before, except that the parameters get permuted: $(\alpha, \beta, \gamma, \delta) \rightarrow$ $(-\beta,-\alpha,-\gamma, \delta)$.

The singularity $w=1$ is different; when it is transformed to $u=\infty$ by $w=$ $u /(u-1)$ we get the equation

$$
\begin{aligned}
u^{\prime \prime}= & \left(\frac{1}{2 u}+\frac{1}{2(u-1)}\right) u^{2}-\frac{1}{z} u^{\prime} \\
& -\alpha \frac{u}{z^{2}(u-1)}-\beta \frac{(u-1)}{z^{2} u}-\gamma \frac{u(u-1)}{z}-\delta u(u-1)(2 u-1)
\end{aligned}
$$

which has the expansion

$$
u= \pm \frac{i / \sqrt{2 \delta}}{z-z_{0}}-\frac{ \pm i \sqrt{2 \delta}+\gamma-2 \delta z_{0}}{4 \delta z_{0}}+c\left(z-z_{0}\right)+\cdots
$$

(Our previous polynomial methods [2] could only handle this last expansion.)

For our computer aided search we used the ansatz $P\left(z, w, w^{\prime}\right) / w^{2}(w-1)^{2}$, the new result is as follows:

$$
H_{1}^{\mathrm{V}}=\frac{3 z w^{2}}{2 w(w-1)^{2}}-\frac{(w+1) w^{\prime}}{w(w-1)}-\frac{3 \alpha w}{z}+\frac{3 \beta}{z w}+\frac{3 \gamma(w+1)}{2(w-1)}+\frac{3 \delta z w}{(w-1)^{2}}
$$

At this level $\mathrm{H}_{2}$ is already rather long and not so informative so we do not write it down.

\section{4. $P_{\mathrm{VI}}$}

Finally the sixth Painlevé equation is given by

$$
\begin{aligned}
w^{\prime \prime}= & \frac{1}{2}\left(\frac{1}{w}+\frac{1}{w-1}+\frac{1}{w-z}\right) w^{2}-\left(\frac{1}{z}+\frac{1}{z-1}+\frac{1}{w-z}\right) w^{\prime} \\
& +\frac{w(w-1)(w-z)}{z^{2}(z-1)^{2}}\left(\alpha+\beta \frac{z}{w^{2}}+\gamma \frac{z-1}{(w-1)^{2}}+\delta \frac{z(z-1)}{(w-z)^{2}}\right) .
\end{aligned}
$$

It has four kinds of singularities: $w=0,1, z, \infty$. The first three can be transformed to infinity using the transformations $w=z / u, w=(u-z) /(u-1), w=z(u-1) /(u-z)$. The resulting equation is as in (20) except for the corresponding changes in the 
parameters: $(\alpha, \beta, \gamma, \delta) \rightarrow(-\beta,-\alpha,-\delta+1 / 2,-\gamma+1 / 2)$ or $(\gamma, \delta-1 / 2, \alpha, \beta+1 / 2)$ or $(-\delta+1 / 2,-\gamma,-\beta,-\alpha+1 / 2)$. At each singularity the expansion is, after the transformation,

$$
w= \pm \frac{\left(z_{0}-1\right) z_{0} / \sqrt{2 \alpha}}{z-z_{0}}+O(1)
$$

with proper replacement for $\alpha$. For the search of $H_{i}^{\mathrm{VI}}$ we used an ansatz with the denominator $w^{2}(w-1)^{2}(w-z)^{2}$. The result is

$$
\begin{aligned}
H_{1}^{\mathrm{VI}=} & 2\left(\frac{z-1}{w}-\frac{z}{w-1}+\frac{1}{w-z}\right) w^{2}+\left(\frac{1}{w}+\frac{1}{w-1}-\frac{3}{w-z}\right) w^{\prime} \\
& -\frac{4 \alpha w}{z(z-1)}+\frac{4 \beta}{w(z-1)}+\frac{4 \gamma w}{z(w-1)}+\frac{4 \delta}{w-z}-\frac{1}{w-z} .
\end{aligned}
$$

(If we were to add the $w$-independent term $2(\alpha-\beta-\gamma+\delta+1 / 2) /(z-1)$ to $H_{1}^{\mathrm{VI}}$ it would be invariant under the transformations above.)

The expression given in (3) of [5] is

$$
u:=\frac{1}{4}\left[H_{1}^{\mathrm{VI}}-\frac{d}{d z}[\log w(w-1)(w-z)]\right]
$$

and yields

$$
e^{\int u d z}=\left[\frac{z-z_{0}+\cdots}{w(w-1)(w-z)}\right]^{1 / 4},
$$

which also has a regular power series expansion at each type of movable singularity.

\section{Summary}

For each Painlevé equation we have constructed expressions $H_{n}\left(z, w, w^{\prime}\right), n=1,2$, quadratic in $w^{\prime}$ and rational in $w, z$, such that

$$
H_{1} \sim \frac{1}{\left(z-z_{0}\right)}+\mathrm{const}, \quad H_{2} \sim \frac{1}{\left(z-z_{0}\right)^{2}}+\text { const }
$$

at each type of movable singularity. Furthermore $\frac{d}{d z} H_{1}+H_{2}$ is always proportional to the corresponding Painlevé equation. If we define

$$
F=e^{\int H_{1} d z}=e^{\iint H_{2} d z d z}, \quad G=w F
$$

then these functions are regular at each kind of movable singularity and satisfy the pair of equations (8),(9). 
It should be mentioned that the Hamiltonians given for the Painlevé equations by Okamoto[3] are somewhat similar and using them one can also construct entire functions. One difference is, however, that our functions $H$ have identical behaviour at all singularities, while Okamoto's Hamiltonians either have a pole $1 /\left(z-z_{0}\right)$ or are regular at the movable singularity, depending, for example, on the sign of the residue.

Finally we note that since $H_{1}$ is a nice meromorphic function one may ask if it solves some interesting equation. Indeed if we define $Y=H_{1}^{\mathrm{I}}$ then $Y$ solves

$$
\left(Y^{\prime \prime}\right)^{2}+4 Y^{\prime 3}+2\left(z Y^{\prime}-Y\right)=0,
$$

and if $Y=H_{1}^{\text {II }}$ we get

$$
\left[\left(Y^{\prime \prime}\right)^{2}+4 Y^{\prime}\left(Y^{\prime 2}-z Y^{\prime}+Y\right)\right]^{2}+64 \alpha^{2} Y^{\prime 3}=0 .
$$

Similar equations should be obtainable for the higher Painlevé equations as well.

\section{Acknowledgments}

I would like to thank M. Kruskal, C. Cosgrove, N. Joshi and N. Witte for discussions.

\section{References}

[1] J. Hietarinta, "Painlevé equations in terms of entire functions", in The Painlevé property: One century later (ed. R. Conte), (Springer, New York, 1999) 661-686.

[2] J. Hietarinta and M. Kruskal, "Hirota forms for the six Painlevé equations from singularity analysis", in Painlevé Transcendent (eds. D. Levi and P. Winternitz), (Plenum Press, New York, 1992) 175-185.

[3] K. Okamoto, "On the $\tau$-function of the Painlevé equations", Physica D 2 (1980) 525-535.

[4] P. Painlevé, "Sur les équations différentielles du second ordre à points critiques fixes", C.R. Acad. Sci. Paris 126 (1898) 1697-1700;

[5] P. Painlevé, "Sur les équations différentielles du second ordre à points critiques fixes", C.R. Acad. Sci. Paris 143 (1906) 1111-1117.

[6] J. Springael, "Direct combinatorial schemes for the application of the Hirota method in soliton theory", Ph.D. Thesis, Vrije Universiteit Brussels, 1999. 